\title{
Pathways of 2,4,6-Trinitrotoluene Transformation by Aerobic Yeasts
}

\author{
Ayrat M. Ziganshin and Robin Gerlach
}

\section{Introduction}

The production and use of various highly persistent synthetic compounds lead to environmental pollution. Among such compounds, 2,4,6-trinitrotoluene (TNT) is the one which is commonly used as an explosive. Synthesis and wide use of TNT in ammunition have resulted in the contamination of soil, air, surface water, and groundwater. TNT and its nitro group reduction products are highly toxic, potentially mutagenic and persistent contaminants which can persist in the environment for a long time (Spain et al. 2000; Stenuit et al. 2005; Smets et al. 2007; Singh et al. 2012). The U.S. Environmental Protection Agency has classified TNT as one of the most dangerous pollutants in the biosphere. Hence, remediation of TNT-contaminated sites is urgently warranted at places of its production and use (Keith and Telliard 1979; Fiorella and Spain 1997).

Human exposure to TNT or its nitro group reduction metabolites can lead to the development of diseases, such as aplastic anemia, cataracts, impaired liver function and the formation of tumors in the urinary tract (Hathaway 1985; Yinon 1990; Leung et al. 1995). Hence, it is inevitable to work out strategies targeting the degradation of TNT.

Decontamination of sites contaminated with explosives, especially with TNT, is possible with application of various physical, chemical, and biological methods. The main advantages of bioremediation are environmental friendliness and involvement of low cost (Rodgers and Bunce 2001).

\footnotetext{
A. M. Ziganshin ( $ه)$

Department of Microbiology, Kazan (Volga Region) Federal University, ul. Kremlyovskaya 18, Kazan, The Republic of Tatarstan, Russia, 420008,

e-mail: a.ziganshin06@fulbrightmail.org

R. Gerlach

Center for Biofilm Engineering and Department of Chemical and Biological Engineering, Montana State University, Bozeman, MT 59717, USA
} 
Since 1980, a wide range of TNT transformation pathways have been worked out. The reductive transformation of the nitro groups appears to be the most commonly observed biologically mediated transformation of TNT (Michels and Gottschalk 1994; Naumov et al. 1999; Huang et al. 2000; Borch et al. 2005). This transformation proceeds via nitroso-dinitrotoluenes (NSDNTs) and hydroxylamino-dinitrotoluenes (HADNTs) with latter being a meta-stable and easily detectable intermediate on the path to complete nitro group to amino group reduction. However, the school of Prof. H.J. Knackmuss focused on the discovery of alternative TNT biotransformation pathways (Vorbeck et al. 1994, 1998). Some microorganisms were found to perform TNT transformation via hydride ion addition to the aromatic ring. This TNT transformation pathway was first described by Vorbeck et al. (1994) for Mycobacterium sp. strain HL 4-NT-1 which leads to the formation of a C-3 monohydride-Meisenheimer complex of TNT $\left(3-\mathrm{H}^{-}-\right.$ TNT). Further transformation of $3-\mathrm{H}^{-}$TNT leads to the accumulation of a C-3,C-5 dihydride-Meisenheimer complex of TNT $\left(3,5-2 \mathrm{H}^{-}-\mathrm{TNT}\right)$, which can be protonated to form $3,5-2 \mathrm{H}^{-} \mathrm{TNT} \cdot \mathrm{H}^{+}$. Three different isomers of $3,5-2 \mathrm{H}^{-}-\mathrm{TNT} \cdot \mathrm{H}^{+}$ were identified. Subsequent studies have demonstrated that other microorganisms are also able to reduce the aromatic ring of TNT by hydride ion attack (French et al. 1998; Kim and Song 2000; Pak et al. 2000; Zaripov et al. 2002; Jain et al. 2004; Wittich et al. 2008; Ziganshin et al. 2010a, b).

TNT nitro group reduction by microbial enzyme systems can lead to the accumulation of highly toxic nitroso- and hydroxylamino-dinitrotoluenes (Leung et al. 1995; Zaripov et al. 2002). TNT aromatic ring attack by hydride ions can cause transformation of TNT with the release of nitrogen in the form of $\mathrm{NO}_{2}{ }^{-}$. In several works, denitration was suggested to be the result of the destruction of TNT-hydride complexes (French et al. 1998; Jain et al. 2004; Williams et al. 2004; Ziganshin et al. 2007, 2010a, b). Other workers suggest that the elimination of a nitro group can occur not only via the hydride pathway, but also during reactions (including abiotic reactions) of HADNTs with Meisenheimer dihydride complexes, producing amino-dimethyl-tetranitrobiphenyls or secondary diarylamines (Pak et al. 2000; Wittich et al. 2008).

Obviously, the use of microorganisms for TNT reduction via hydride ion addition and subsequent degradation of the formed complexes is highly promising from a viewpoint of bioremediation of TNT-contaminated sites.

The ability to detect metabolites of TNT transformation via alternative pathways is very important from the viewpoint of evaluating the effectiveness of TNTcontaminated site remediation. Improvement in methods for detecting TNT transformation intermediates certainly contributes to our understanding of transformation mechanisms and thus helps in better controlling TNT degradation. The use of improved HPLC-MS methods for separation of TNT transformation products helped in the identification of carbon containing metabolites produced via nitro group reduction as well as via aromatic ring reduction in a single HPLC run, therefore minimizing the potential for changes in sample composition after sampling (Ziganshin et al. 2007, 2010a, b). This had not been possible in most of the previous works investigating the aromatic ring reduction of TNT by 
microorganisms (French et al. 1998; Pak et al. 2000; Jain et al. 2004; Williams et al. 2004; Wittich et al. 2008).

As a result, new insights into the mechanism of TNT transformation by Yarrowia lipolytica AN-L15 and Geotrichum sp. AN-Z4 (closely related to Geotrichum candidum) have been made which indicate interesting interactions and interplays of biological, chemical and physical parameters affecting TNT transformation.

Aromatic ring as well as nitro group reduction products were monitored and detected along with nitrite, nitrate and nitric oxide as transformation products. An improved understanding and control of the transformation pathway should enhance the effectiveness of technologies for the bioremediation of TNT-contaminated areas in future.

\section{Pathways of TNT Degradation}

The lack of a complete understanding of the exact mechanisms of TNT reduction mediated by hydride ions and denitration of the formed metabolites prompted us to study this process in more detail. Two newly isolated yeasts were found capable of TNT transformation via hydride ion addition to the TNT aromatic ring (Ziganshin et al. 2007, 2010b). We also established analytical methods which enabled us to study TNT transformation via hydride complexes initiated by these yeasts (Borch and Gerlach 2004; Ziganshin et al. 2007).

The two yeast strains were identified as Yarrowia lipolytica AN-L15 (Ziganshin et al. 2007) and Geotrichum sp. AN-Z4 [closely related to Geotrichum candidum; Ziganshin et al. (2010b)]. Direct aromatic ring reduction via hydride Meisenheimer complex formation was observed as the dominant pathway of TNT transformation by these yeasts along with some TNT nitro group reduction occurring simultaneously.

The aromatic ring reduction of TNT allows the fission of the aromatic rings which opens up the possibility of TNT mineralization. However, only a few strains of bacteria (French et al. 1998; Vorbeck et al. 1998; Pak et al. 2000; Kim et al. 2002; van Dillewijn et al. 2008; Wittich et al. 2008) and fungi (Kim and Song 2000; Zaripov et al. 2002; Jain et al. 2004) were demonstrated to be capable of TNT transformation via aromatic ring reduction.

Since the effectiveness of remediation technologies for TNT-contaminated areas depends on the effective control of biological and abiotic transformations of TNT, HPLC-based methods were developed for the separation and identification of key intermediates formed during TNT conversion (Borch and Gerlach 2004; Borch et al. 2005; Ziganshin et al. 2007). These methods utilize reversed phase high performance liquid chromatography (HPLC) separation combined with diode-array (DAD) and atmospheric pressure chemical ionization mass spectrometric (APCI-MS) detection. In particular, varying the temperature and composition of chromatographic eluents, the isomers, 2-hydroxylamino-4,6-dinitrotoluene (2-HADNT) and 4-hydroxylamino-2,6-dinitrotoluene (4-HADNT) were separated 
by HPLC. It was a great challenge in previous works (e.g., Michels and Gottschalk 1994; Vorbeck et al. 1998; Hawari et al. 1999; Naumov et al. 1999).

After isolation of the two yeast strains (AN-L15 and AN-Z4), the HPLC methods were used to reliably separate many of the TNT aromatic ring transformation products as well. Hence, these methods were applied to explore the mechanism of TNT aromatic ring reduction by these yeast strains.

The dominant pathway of TNT conversion by the yeasts is based on the addition of hydride ions to the aromatic ring. In this pathway, we demonstrated that the reduction of TNT by hydride ions was associated with the formation of at least eight different mono- and dihydride-Meisenheimer complexes (Figs. 1, 2, Table 1) (Ziganshin et al. 2007, 2010a, b). In earlier studies, researchers had only been able to demonstrate the formation of only five hydride forms of TNT, in

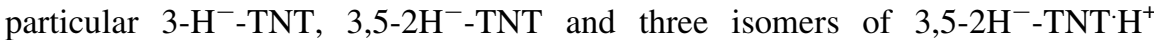
(Vorbeck et al. 1998; Pak et al. 2000; Williams et al. 2004).

Our work detected eight metabolites which were characterized by us as TNThydride complexes based on (1) their UV-visible absorbance spectra, which show strong absorbance maxima between 440 and $500 \mathrm{~nm}$, (2) their mass spectra (Table 1), and (3) observed biotic and abiotic conversion reactions (Ziganshin et al. 2007).

Molecular ions and mass fragments, observed during negative mode APCI-MS mass spectrometry of $3-\mathrm{H}^{-}$-TNT, were similar to those obtained in earlier work (Yinon et al. 1995) with dominant fragments of $\mathrm{m} / z=197,210$, and 227. Mass spectrometric analysis of other TNT-hydride complexes after HPLC-based purification enabled us to divide them into three groups (Table 1):

1. compounds with a molecular ion at $m / z 227$ (compounds 1, 5, 7, and 8), presumably TNT-monohydride complexes;

2. a compound with a molecular ion at $m / z 228$ (compound 4), presumably a TNTdihydride complex;

3. compounds with a molecular ion at $m / z 230$ (compounds 2, 3, and 6), presumably protonated TNT-dihydride complexes.

The existence of three complexes with a molecular mass of 230 was also earlier reported (Vorbeck et al. 1998; Pak et al. 2000) and characterized as 3,5-2 $\mathrm{H}^{-}$TNT $\mathrm{H}^{+}$isomers. Compounds 1 and 5 appear to be additional isomers of $3-\mathrm{H}^{-}-$ TNT (compound 7) and were not described before. They were characterized by us in 2007 (Ziganshin et al. 2007). Besides, we also observed and characterized an apparent C-1 Meisenheimer monohydride complex (1- $\mathrm{H}^{-}-\mathrm{TNT}$, compound 8), which was produced by the yeasts to a much lesser extent than the $3-\mathrm{H}^{-}$-TNT complexes and did not interconvert into any of the other seven detected TNThydride complexes.

The HPLC-method was also utilized in our works for separating 3,5-2 $\mathrm{H}^{-}$-TNT. Previously, the existence of this metabolite was only proposed by Vorbeck et al. (1998). However, we were able to separate it from the $3,5-2 \mathrm{H}^{-}-\mathrm{TNT} \cdot \mathrm{H}^{+}$isomers and obtain its mass and UV-visible absorbance spectra. 

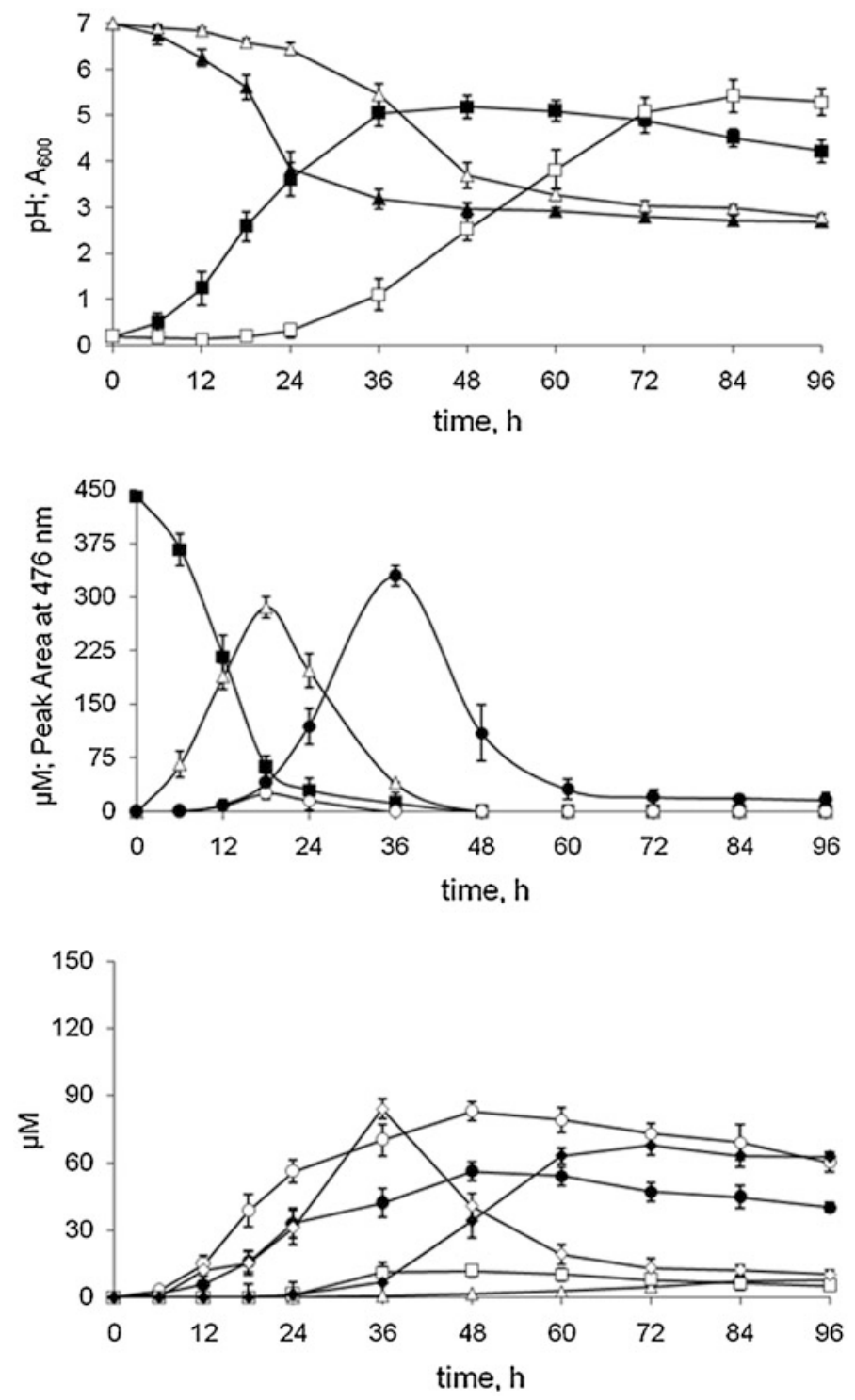

Fig. 1 a Growth $\left(\mathrm{A}_{600}\right)$ of $Y$. lipolytica AN-L15 and subsequent changes in culture medium $\mathrm{pH}$ (initial $\mathrm{pH}$ 7.0). Symbols: filled square, growth $\left(\mathrm{A}_{600}\right)$ and filled triangle, $\mathrm{pH}$ change in the absence of TNT; square, growth $\left(\mathrm{A}_{600}\right)$ and triangle, $\mathrm{pH}$ change in the presence of TNT. b Accumulation of metabolites during TNT transformation by strain AN-L15. Symbols: filled

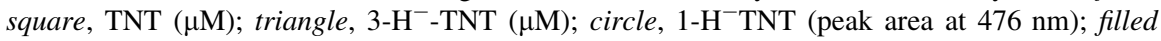
circle, sum of Meisenheimer complexes related to $3-\mathrm{H}^{-}$-TNT (compounds 1-6; peak area at $476 \mathrm{~nm}$; c filled circle, 2-HADNT $(\mu \mathrm{M})$; circle, 4-HADNT $(\mu \mathrm{M})$; diamond, $\mathrm{NO}_{2}{ }^{-}(\mu \mathrm{M})$; filled diamond, $\mathrm{NO}_{3}{ }^{-}(\mu \mathrm{M})$; square, 2,4-DNT $(\mu \mathrm{M})$; triangle, 4-ADNT $(\mu \mathrm{M})$. Error bars indicate the standard deviation of triplicate experiments (Ziganshin et al. 2010a). TNT transformation by Geotrichum sp. AN-Z4 was similar to TNT transformation by Y. lipolytica AN-L15 and is not shown. Reprinted with permission from Ziganshin et al.(2010a) 


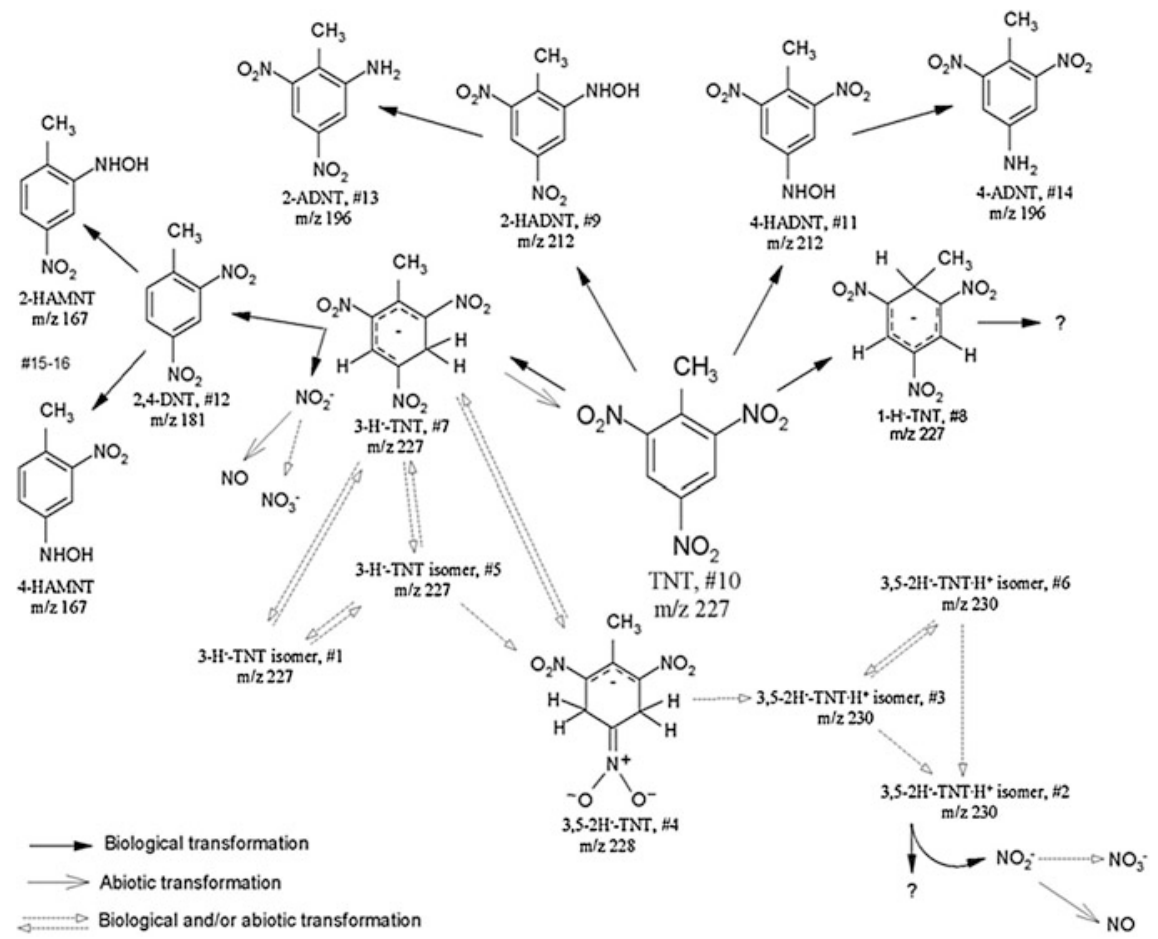

Fig. 2 Proposed pathways of TNT transformation in the presence of $Y$. lipolytica AN-L15 and Geotrichum sp. AN-Z4. Data obtained during our research did not allow structural distinction between the different $3-\mathrm{H}^{-}$-TNT or $3,5-2 \mathrm{H}^{-}-\mathrm{TNT} \cdot \mathrm{H}^{+}$isomers

The ability to separate and purify these TNT aromatic ring reduction products allowed us to explore the stability and conversion of individual TNT-hydride complexes under various physicochemical conditions. Such studies also enabled us to estimate the relative importance of biologically and physico-chemically dominated transformation reactions of these compounds.

Previously TNT was detected as the only product of the spontaneous abiotic transformation of (chemically synthesized) 3- $\mathrm{H}^{-}-\mathrm{TNT}$ (Vorbeck et al. 1998; Pak et al. 2000). However, our work demonstrated the possibility of $3-\mathrm{H}^{-}-\mathrm{TNT}$ to be abiotically converted into its isomers (compounds 1 and 5), TNT as well as 3,5$2 \mathrm{H}^{-}$-TNT (compound 4). Besides, we also showed the possibility of five more TNT-hydride complexes (except for compound 2, a 3,5-2 $\mathrm{H}^{-}-\mathrm{TNT} \cdot \mathrm{H}^{+}$isomer, and $\left.1-\mathrm{H}^{-}-\mathrm{TNT}\right)$ to be interconverted. All these abiotic reactions proceed without the elimination of nitro groups.

Enzymatic production of $1-\mathrm{H}^{-}$-TNT from TNT was earlier proposed. French et al. (1998) suggested the production of $1-\mathrm{H}^{-}$-TNT by pentaerythritol tetranitrate reductase isolated from Enterobacter cloacae $\mathrm{PB}$. They observed predominantly 3$\mathrm{H}^{-}$-TNT as the product of the initial hydride-ion mediated attack on TNT. Besides, 
Table 1 Metabolites formed during transformation of TNT by yeasts under various cultivation conditions

\begin{tabular}{lllll}
\hline S1 no. & Compound & Molecular ion $^{\mathrm{a}}$ & $\mathrm{RT}$ at 36 and $50{ }^{\circ} \mathrm{C}, \mathrm{min}^{\mathrm{b}}$ & $\lambda_{\max }, \mathrm{nm}^{\mathrm{c}}$ \\
\hline 1 & $3-\mathrm{H}^{-}-\mathrm{TNT}$ isomer & 227 & $4.8 / 4.7$ & 261,445 \\
2 & $3,5-2 \mathrm{H}^{-}-\mathrm{TNT} \cdot \mathrm{H}^{+}$isomer & 230 & $5.2 / 4.9$ & 266,426 \\
3 & $3,5-2 \mathrm{H}^{-}$-TNT $\mathrm{H}^{+}$isomer & 230 & $5.3 / 5.0$ & 263,478 \\
4 & $3,5-2 \mathrm{H}^{-}-\mathrm{TNT}$ & 228 & $5.7 / 5.4$ & 325,512 \\
5 & $3-\mathrm{H}^{-}-\mathrm{TNT}$ isomer & 227 & $5.9 / 5.5$ & 262,465 \\
6 & $3,5-2 \mathrm{H}^{-}-\mathrm{TNT} \cdot \mathrm{H}^{+}$isomer & 230 & $6.7 / 6.2$ & 263,491 \\
7 & $3-\mathrm{H}^{-}-\mathrm{TNT}$ isomer & 227 & $9.9 / 8.5$ & $256,480,550$ \\
8 & $1-\mathrm{H}^{-}$-TNT & 227 & $12.3 / 10.3$ & $251,478,551$ \\
9 & 2-HADNT & 212 & $14.8 / 12.4$ & $228,265,356$ \\
10 & TNT & 227 & $15.4 / 13.3$ & 230 \\
11 & 4-HADNT & 212 & $16.0 / 13.3$ & 232,350 \\
12 & $2,4-\mathrm{DNT}$ & 181 & $17.7 / 15.1$ & 250 \\
13 & 2-ADNT & 196 & $17.0 / 14.1$ & $225,270,375$ \\
14 & 4-ADNT & 196 & $17.6 / 14.7$ & 235,362 \\
$15-16$ & HAMNT & 167 & $10.9-11.1 /-$ & - \\
\hline
\end{tabular}

\footnotetext{
${ }^{a}$ Molecular ion detected during negative mode APCI-MS analysis

${ }^{\mathrm{b}}$ HPLC retention times observed at two different separation temperatures

${ }^{\mathrm{c}}$ UV-visible absorbance maxima
}

indication of another metabolite with similarspectral characteristics was described. This second compound was suggested to be $1-\mathrm{H}^{-}-\mathrm{TNT}$, but its production could not be confirmed in the subsequent work (Williams et al. 2004). Based on the UV-visible absorbance and mass spectra along with abiotic interconversion studies, it was concluded that compound 8 must be $1-\mathrm{H}^{-}$-TNT (Ziganshin et al. 2007).

As previously described (Kim and Song 2000; Jain et al. 2004; Williams et al. 2004), the reduction of the aromatic ring of TNT by yeast strains also led to the release of inorganic nitrogen containing compounds. This pathway is very important in view of TNT biodegradation efficiency. Lesser nitrated compounds, such as dinitrotoluenes, are more susceptible to aromatic ring fission than the more stable TNT.

According to our findings, there are at least two different pathways of TNT biodegradation via TNT-hydride complexes (Ziganshin et al. 2007, 2010a, b). One of the pathways leads to the elimination of one nitro group from $3-\mathrm{H}^{-}$-TNT with simultaneous accumulation of nitrite and 2,4-dinitrotoluene (2,4-DNT). The accumulation of the latter metabolite occurred only during the phase of $3-\mathrm{H}^{-}$-TNT disappearance (Figs. 1, 2). However, production of 2,4-DNT was dependent on the $\mathrm{pH}$ of the growth medium, which changed throughout the experiment because of the production of organic acids by the yeasts (Ziganshin et al. 2010a, b).

Yeast strain AN-L15 produced and excreted citrate and pyruvate into the culture medium, while strain AN-Z4 produced citrate, succinate and isocitrate which caused acidification of the medium during their growth. This acidification of the medium also initiated the oxidation of the released nitrite to nitrate. 
The production of dinitrotoluenes from TNT results in a class of compounds, which can more easily undergo aromatic ring fission due to their increased susceptibility to oxidative attack of the aromatic ring compared to TNT (Spanggord et al. 1991).

As reflected in Fig. 1, depending on the culture conditions, a small part of 2,4DNT formed was also converted to hydroxylamino-mononitrotoluenes (HAMNTs) in our experiments, indicating the possibility of nitro group reduction of DNTs by these yeast strains (Ziganshin et al. 2010a).

The second possible pathway of TNT biodegradation by strains AN-L15 and AN-Z4 seems to proceed via transformation of one of the isomers of $3,5-2 \mathrm{H}^{-}$TNT $\mathrm{H}^{+}$(apparently compound 2 in our scheme; Ziganshin et al. 2007). The release of nitrite through this pathway started during the early stages of TNT transformation, even without detectable 2,4-DNT production (Fig. 1). Continued aerobic cultivation of the yeasts led to a decrease in medium $\mathrm{pH}$ and to the oxidation of nitrite into nitrate. The nitrite to nitrate conversion occurred enzymatically as well as abiotically once a low enough $\mathrm{pH}$ was attained. Nitric oxide (NO) production was also observed and attributed to an abiotically occurring disproportionation reaction, which converts nitrite to nitrate and nitric oxide (Ziganshin et al. 2010a; Khilyas et al. 2013).

Williams et al. (2004), in their experiments with pentaerythritol tetranitrate reductase of E. cloacae $\mathrm{PB}$, suggested that the source of nitrite is one of the isomers of $3,5-2 \mathrm{H}^{-}-\mathrm{TNT} \cdot \mathrm{H}^{+}$(possibly compound 2 in our scheme). The suggestion of Pak et al. (2000) regarding the possible role of isomers of amino-dimethyltetranitrobiphenyls in nitrite release could not be confirmed by us, since these compounds were not detected in any of our experiments. Furthermore, diarylamines, which were formed through condensation of 3,5-2 $\mathrm{H}^{-}$-TNT with HADNTs according to Wittich et al. (2008), were not identified either. Instead, the production of nitrite indicates the possibility of aromatic ring fission and therefore, the possibility of TNT mineralization.

However, as mentioned above, TNT transformation by the yeast strains Y. lipolytica AN-L15 and Geotrichum sp. AN-Z4 did not solely proceed via TNT-hydride complexes, but also led to the reduction of nitro groups resulting in the formation of HADNTs and ADNTs (Figs. 1, 2, Table 1). Retention times, mass and UV-visible spectra of these nitro group reduction products are consistent with those obtained during the analysis of chemical standards. However, the extent of nitro group reduction by strains AN-L15 and AN-Z4 was less common relative to the aromatic ring reduction pathway described above. Nevertheless, as it has been observed previously (e.g. Hawari et al. 1998; Borch et al. 2005), nitro group reduction preferentially (though not exclusively) occurred in the para position leading to higher concentrations of 4-HADNT and 4-ADNT than of 2-HADNT and 2-ADNT. 


\section{Conclusions}

Despite several studies on the mechanisms of TNT transformation by organisms of different evolutionary levels, the understanding of TNT biotransformation is still far from complete. An improved understanding of the degradation pathways depends on improved methods for the detection of intermediates formed during transformation.

The application of a new HPLC-diode array/mass spectrometric detection method has over the past years helped in improved separation of different peaks which facilitated the identification of novel intermediates of TNT transformation. The obligate aerobic yeast strains $Y$. lipolytica AN-L15 and Geotrichum sp. ANZ4 transform TNT via two principally different pathways: (1) aromatic ring reduction as the primary transformation pathway resulting in TNT-hydride complexes as intermediates and ultimately leading to the elimination of nitro groups from the aromatic systems as well as the possible destruction of the TNT aromatic backbone; and (2) nitro group reduction leading to the production of hydroxylamino- and amino-dinitrotoluenes. Eight different TNT-hydride complexes were identified and characterized as follows: (1) Compounds with molecular ions with mass to charge ratios $(\mathrm{m} / \mathrm{z})$ of 227 are TNT-mono-hydride complexes $\left(1-\mathrm{H}^{-}\right.$-TNT and $3-\mathrm{H}^{-}$-TNT, of which we identified three different isomers); (2) a compound with $m / z=228$ is a TNT-dihydride complex (3,5-2 $\mathrm{H}^{-}$-TNT); and (3) compounds with $m / z=230$ are protonated TNT-dihydride complexes (three isomers of 3,5$2 \mathrm{H}^{-}$-TNT $\mathrm{H}^{+}$detected by us).

Our studies not only demonstrated the ability of these yeast strains to produce TNT-hydride-Meisenheimer complexes, but also to facilitate the elimination of nitro groups in the form of nitrite and nitrate.

There appear to be at least two principally different pathways of nitro group elimination from the produced Meisenheimer complexes: one pathway is based on the decomposition of $3-\mathrm{H}^{-}$-TNT with the formation of 2,4-DNT. Another pathway is based on the degradation of one of the isomers of $3,5-2 \mathrm{H}^{-}-\mathrm{TNT} \cdot \mathrm{H}^{+}$. It is also possible that a third pathway exists that involves $1-\mathrm{H}^{-}-\mathrm{TNT}$ as an intermediate, but due to the low amounts of $1-\mathrm{H}^{-}$-TNT produced by the yeasts, it has not been possible to purify sufficient amounts of $1-\mathrm{H}^{-}$-TNT to demonstrate the possible pathway.

Each one of these pathways results in the release of the nitro-groups from the carbon skeleton of TNT, accompanied by the appearance of nitrite, which can be further converted enzymatically to nitrate and abiotically to nitrate and NO.

The yeasts Y. lipolytica AN-L15 and Geotrichum sp. AN-Z4 were isolated from oil-polluted peat bog and petrochemical wastes, respectively. Their ability to survive under such extreme conditions, combined with their fairly unique mechanism of TNT degradation, makes these microorganisms promising for the bioremediation of soils and industrial wastes contaminated with explosives and potentially other (nitro)aromatic compounds. 
Acknowledgments This chapter is dedicated to John Neuman († Feb 20, 2011), Laboratory Manager at the Center for Biofilm Engineering at Montana State University from 1994 to 2008. His indispensable help with all aspects of analytical chemistry, proper laboratory techniques, safety and 'life in general' is gratefully acknowledged and will be forever remembered.

This work was supported by the Fulbright Program. Partial financial support was provided by the US Department of Defense, Army Research Office, Grant No. DAAD19-03-C-0103 and the Office of Science (BER), U.S. Department of Energy, Grant No. DE-FG-02-09ER64758.

\section{References}

Borch T, Gerlach R (2004) Use of reversed-phase high-performance liquid chromatographydiode array detection for complete separation of 2,4,6-trinitrotoluene metabolites and EPA method 8,330 explosives: influence of temperature and an ion-pair reagent. J Chromatogr A 1022:83-94

Borch T, Inskeep WP, Harwood JA, Gerlach R (2005) Impact of ferrihydrite and anthraquinone2,6-disulfonate on the reductive transformation of 2,4,6-trinitrotoluene by a gram-positive fermenting bacterium. Environ Sci Technol 39:7126-7133

Fiorella PD, Spain JC (1997) Transformation of 2,4,6-trinitrotoluene by Pseudomonas pseudoalcaligenes JS52. Appl Environ Microbiol 63:2007-2015

French CE, Nicklin S, Bruce NC (1998) Aerobic degradation of 2,4,6-trinitrotoluene by Enterobacter cloacae $\mathrm{PB} 2$ and by pentaerythritol tetranitrate reductase. Appl Environ Microbiol 64:2864-2868

Hathaway JA (1985) Subclinical effects of trinitrotoluene: a review of epidemiology studies. In: Rickert DE (ed) Toxicity of nitroaromatic compounds hemisphere publishing corporation Washington DC, pp 255-274

Hawari J, Halasz A, Beaudet S, Paquet L, Ampleman G, Thiboutot S (1999) Biotransformation of 2,4,6-trinitrotoluene with Phanerochaete chrysosporium in agitated cultures at $\mathrm{pH} 4.5$. Appl Environ Microbiol 65:2977-2986

Hawari J, Halasz A, Paquet L, Zhou E, Spencer B, Ampleman G, Thiboutot S (1998) Characterization of metabolites in the biotransformation of 2,4,6-trinitrotoluene with anaerobic sludge: role of triaminotoluene. Appl Environ Microbiol 64:2200-2206

Huang S, Lindahl PA, Wang C, Bennett GN, Rudolph FB, Hughes JB (2000) 2,4,6Trinitrotoluene reduction by carbon monoxide dehydrogenase from Clostridium thermoaceticum. Appl Environ Microbiol 66:1474-1478

Jain MR, Zinjarde SS, Deobagkar DD, Deobagkar DN (2004) 2,4,6-Trinitrotoluene transformation by a tropical marine yeast, Yarrowia lipolytica NCIM 3589. Marine Pollut Bull 49:783-788

Keith LH, Telliard WA (1979) Priority pollutants: a perspective view. Environ Sci Technol 13:416-423

Khilyas IV, Ziganshin AM, Pannier AJ, Gerlach R (2013) Effect of ferrihydrite on 2,4,6trinitrotoluene biodegradation by an aerobic yeast. Biodegradation. doi:10.1007/s10532-0129611-4

Kim H-Y, Bennett GN, Song H-G (2002) Degradation of 2,4,6-trinitrotoluene by Klebsiella sp. isolated from activated sludge. Biotechnol Lett 24:2023-2028

Kim H-Y, Song H-G (2000) Comparison of 2,4,6-trinitrotoluene degradation by seven strains of white rot fungi. Curr Microbiol 41:317-320

Leung KH, Yao M, Stearns R, Chiu S-HL (1995) Mechanism of bioactivation and covalent binding of 2,4,6-trinitrotoluene. Chem Biol Interact 97:37-51 
Michels J, Gottschalk G (1994) Inhibition of the lignin peroxidase of Phanerochaete chrysosporium by hydroxylamino-dinitrotoluene, an early intermediate in the degradation of 2,4,6-trinitrotoluene. Appl Environ Microbiol 60:187-194

Naumov AV, Suvorova ES, Boronin AM, Zaripova SK, Naumova RP (1999) Transformation of 2,4,6-trinitrotoluene into toxic hydroxylamino derivatives by Lactobacilli. Microbiology 68:56-62 (Russia)

Pak JW, Knoke KL, Noguera DR, Fox BG, Chambliss GH (2000) Transformation of 2,4,6trinitrotoluene by purified xenobiotic reductase B from Pseudomonas fluorescens I-C. Appl Environ Microbiol 66:4742-4750

Rodgers JD, Bunce NJ (2001) Treatment methods for the remediation of nitroaromatic explosives. Water Res 35:2101-2111

Singh B, Kaur J, Singh K (2012) Microbial remediation of explosive waste. Crit Rev Microbiol 38:152-167

Smets BF, Yin H, Esteve-Núñez A (2007) TNT biotransformation: when chemistry confronts mineralization. Appl Microbiol Biotechnol 76:267-277

Spain JC, Hughes JB, Knackmuss H-J (2000) Biodegradation of nitroaromatic compounds and explosives. Lewis Publishers, Boca Raton

Spanggord RJ, Spain JC, Nishino SF, Mortelmans KE (1991) Biodegradation of 2,4dinitrotoluene by a Pseudomonas sp. Appl Environ Microbiol 57:3200-3205

Stenuit B, Eyers L, Fantroussi SE, Agathos SN (2005) Promising strategies for the mineralization of 2,4,6-trinitrotoluene. Rev Environ Sci Bio/Tech 4:39-60

van Dillewijn P, Wittich RM, Caballero A, Ramos JL (2008) Type II hydride transferases from different microorganisms yield nitrite and diarylamines from polynitroaromatic compounds. Appl Environ Microbiol 74:6820-6823

Vorbeck C, Lenke H, Fischer P, Knackmuss H-J (1994) Identification of a hydride-Meisenheimer complex as a metabolite of 2,4,6-trinitrotoluene by a Mycobacterium strain. J Bacteriol 176:932-934

Vorbeck C, Lenke H, Fischer P, Spain JC, Knackmuss H-J (1998) Initial reductive reactions in aerobic microbial metabolism of 2,4,6-trinitrotoluene. Appl Environ Microbiol 64:246-252

Williams RE, Rathbone DA, Scrutton NS, Bruce NC (2004) Biotransformation of explosives by the old yellow enzyme family of flavoproteins. Appl Environ Microbiol 70:3566-3574

Wittich RM, Haidour A, van Dillewijn P, Ramos JL (2008) OYE flavoprotein reductases initiate the condensation of TNT-derived intermediates to secondary diarylamines and nitrite. Environ Sci Technol 42:734-739

Yinon J (1990) Toxicity and metabolism of explosives. CRC Press, Boca Raton

Yinon J, Johnson JV, Bernier UR, Yost RA, Mayfield HT, Mahone WC, Vorbeck C (1995) Reactions in the mass spectrometry of a hydride meisenheimer complex of 2,4,6trinitrotoluene (TNT). J Mass Spectrom 30:715-722

Zaripov SA, Naumov AV, Abdrakhmanova JF, Garusov AV, Naumova RP (2002) Models of 2,4,6-trinitrotoluene (TNT) initial conversion by yeasts. FEMS Microbiol Lett 217:213-217

Ziganshin AM, Gerlach R, Borch T, Naumov AV, Naumova RP (2007) Production of eight different hydride complexes and nitrite release from 2,4,6-trinitrotoluene by Yarrowia lipolytica. Appl Environ Microbiol 73:7898-7905

Ziganshin AM, Naumova RP, Pannier AJ, Gerlach R (2010a) Influence of pH on 2,4,6trinitrotoluene degradation by Yarrowia lipolytica. Chemosphere 79:426-433

Ziganshin AM, Gerlach R, Naumenko EA, Naumova RP (2010b) Aerobic degradation of 2,4,6trinitrotoluene by the yeast strain Geotrichum candidum AN-Z4. Microbiology 79:199-205 (Russia) 